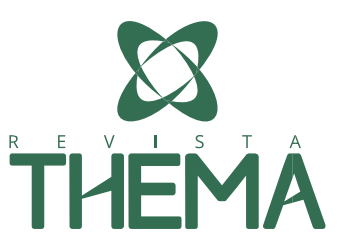

\title{
As aparências se atraem: valores culturais e consumo de produtos de moda falsificados
}

\section{Appearances attract: cultural values and consumption of counterfeit fashion products}

Francisco Nunes dos Reis Junior ${ }^{1}$; Cláudio Torres ${ }^{2}$

\section{RESUMO}

Compreender as diferenças culturais tornou-se crucial para uma comunicação eficaz com o consumidor uma vez que estudos comprovam que a cultura exerce influência na escolha das pessoas por produtos. Este artigo, por meio de uma pesquisa bibliográfica na área de valores culturais, busca explicações para o fenômeno do consumo de produtos de moda falsificados. Como resultado, verificou-se que o valor que melhor pode ajudar na explicação deste tipo de consumo é o individualismo/coletivismo vertical, pois tem ênfase no apreço pela hierarquia social.

Palavras-chave: valores culturais, falsificações, individualismo-coletivismo.

\begin{abstract}
Understanding cultural differences has become crucial for effective communication with consumers as studies have shown that culture influences people's choice for products. This paper, through a bibliographical research in the area of cultural values, seeks explanations for the phenomenon of consumption of counterfeit fashion products. As a result, it was found that the value that may best explain this type of consumption is vertical individualism/collectivism, as it emphasizes the appreciation of the social hierarchy.
\end{abstract}

Keywords: cultural values, counterfeit, individualism-collectivism.

\section{INTRODUÇÃO}

Os seres humanos são uma espécie cultural, dependem criticamente da aprendizagem cultural em praticamente todos os aspectos da vida. Psicólogos culturais compartilham o pressuposto de que nem todos os processos psicológicos são tão armazenados no cérebro que apareçam de forma idêntica em todos os contextos culturais. Em vez disso, os processos psicológicos são vistos como em sintonia com o sistema de significado cultural particular em que o indivíduo se desenvolve. Assim, se estamos interessados em avaliar a universalidade de um fenômeno particular, é necessário analisar os dados de uma grande variedade de amostras. Corroborando com o exposto, Heine (2010) defende que

\footnotetext{
${ }^{1}$ IFB - Instituto Federal de Educação, Ciência e Tecnologia de Brasília, Brasília/DF - Brasil

2 UnB - Universidade de Brasília, Brasília/DF - Brasil.
} 
aprender sobre o funcionamento de outras culturas aumenta o entendimento sobre a nossa própria cultura.

Definir de cultura não é per si uma incursão fácil. Por se tratar de um conceito multidisciplinar, existem esforços de várias áreas das ciências humanas e sociais neste processo de definição, a saber: antropologia cultural, sociologia, psicologia social, psicologia sócio histórica, psicologia clínica, história, comunicação, administração e economia. Neste sentido, cada uma dessas áreas utiliza enfoques diferentes para definir cultura, bem como metodologias próprias na condução das pesquisas. A intenção deste artigo não é avaliar a melhor conceituação teórica e nem metodológica para o estudo da cultura, mas sim utilizar-se do arcabouço teórico que julga ser mais pertinente para analisar o tema proposto.

Das várias definições possíveis, a que parece adequada para o escopo deste trabalho vem da psicologia social e é a proposta por Rohner (1984) onde cultura seria a totalidade dos significados equivalentes e complementares aprendidos e mantidos por uma população humana, ou por segmentos identificáveis de uma população, e transmitida de geração em geração.

Portanto a função dos estudos culturais seria compreender e comparar culturas (sem julgamento de valores) por meio do levantamento de valores, crenças e a ocorrência diferenciais de comportamentos. Para levantar tais dados, as pesquisas podem focar tanto no nível de análise individual quanto no nível de análise nacional. Os estudos em nível individual são pesquisas em que os dados de cada entrevistado são tratados como casos separados (SMITH et al, 2013). Já nos estudos em nível nacional a pesquisa deverá inicialmente fazer a média entre todos os dados de cada nação em cada variável e só depois analisá-los, ao fazer isso tratam cada nação como único caso (SMITH et al, 2013). Para os psicólogos sociais essa diferenciação de níveis é importante não por entender que os indivíduos e as nações são diferentes, mas para que o planejamento das pesquisas, análise de dados e comparações sejam feitas de forma correta: indivíduo com indivíduo e nação versus nação.

Exemplo de pesquisa realizada no nível nacional foi a pesquisa liderada por Hofstede (1984) ao comparar países ao redor no mundo. Por outro lado, o modelo do levantamento de valores proposto por Schwartz (1992) é focado, a priori, no nível de análise individual. Importante ressaltar que estudos de nível nacional podem não levar em consideração as diferenças étnicas de um país, pois ao agruparem-se todos os grupos étnicos sob a égide de uma cultura única, podem-se negligenciar fatores importantes de grupos étnicos específicos (SMITH, BOND \& KAGITÇIBASI, 2006). Este trabalho considerará os valores a nível cultural. No que tange à relação cultura e consumo, a maioria dos modelos culturais não foi desenvolvido para a análise do comportamento consumidor. De Mooij e Hofstede (2011) recomendam que ao usá-los, as manifestações da cultura que são relevantes para o comportamento do consumidor têm que ser selecionadas e interpretadas.

Este artigo junta esforços para explicar teoricamente que o consumo de falsificações de produtos de moda é reflexo, fortemente, da cultura (MOOIJ \& HOFSTEDE, 2011) e que pode ser mais bem explicado pelo viés cultural do individualismo/coletivismo vertical (TRIANDIS \& GELFAND, 1998) onde o consumo (original ou falsificado) funcionaria como mecanismo de gerenciamento de impressão ao invés de um fenômeno com explicação objetiva apenas pelo cunho econômico (GROSSMAN \& SHAPIRO, 1988; JACOBY \& KAPLAN, 1972), legal (CHISTOVÃO, 2001; GRANSTRAND, 2005) ou mercadológico (DELENER, 2000; GENTRY, 2002; ZAICHKOWSKY, 1995). 
Como se percebe, existem esforços em explicar o consumo de falsificações por pontos de vistas diferentes (direito, marketing e economia), mas poucos esforços são estudados no âmbito da psicologia e da moda utilizando conceitos pertinentes das referidas áreas (SHAVITT, TORELLI E WONG, 2009). Por esta razão este artigo buscará arcabouços teóricos da psicologia social, mas que sejam aplicáveis ao estudo da moda na busca de explicações para o fenômeno do consumo de produtos de moda falsificados.

\section{VALORES CULTURAIS}

O estudo dos valores não é recente. A importância desse constructo já era debatida pelos filósofos pré-socráticos, mas só saem do âmbito da filosofia e passam a ser estudados no escopo da ciência com os esforços de Talcott Parsons na publicação do livro a estrutura da ação social em 1937. Importante ainda ressaltar os estudos clássicos de Kluckhohn (1951), Rokeach (1973) e Schwartz (1992). Os autores são unânimes ao afirmar que todo ser social tem valores, que são universais e não podem ser falseados. Por esta razão é um campo em ebulição e com avanços tanto teóricos quanto metodológicos e com aplicações em diferentes áreas de pesquisa (ex: psicologia do consumidor, psicologia das organizações e trabalho, psicologia política).

Valores podem ser definidos como um constructo motivacional que transcendem situações e ações específicas, guiam a seleção e avaliação de ações, políticas, pessoas, eventos e são ordenados pela importância relativa aos demais. A partir desta definição pode-se perceber que os valores são esquemas e, portanto, não são inatos e sim aprendidos pelo processo de generalização. Assim, os valores levam a formação de atitudes e estas levam a comportamentos. Por esta razão, da mesma forma que as atitudes não são boas preditoras de comportamentos específicos (AJZEN, 2008), os valores também não o são. Assim, valores são prescritivos (informam como as coisas devem ser) e não descritivos.

Não se deve confundir valores com normas. Normas são costumes habituais exibidos por um grupo, por servirem para alcançar as necessidades básicas (SUMNER, 1906), são as regras do comportamento social que são negociadas (SHERIF, 1936). A relação a ser guardada é que a norma é o padrão desejado por um grupo e os valores representam o compromisso com essas normas.

Os valores podem ser analisados sobre duas perspectivas: agrupamento de valores e nível de análise. A primeira perspectiva estuda conforme o sistema de valores, os tipos motivacionais e a hierarquia dos valores. Já a segunda, foca em entender os valores em nível micro (individual), meso (grupos) e macro (culturais). Esta segunda perspectiva é a que será abordada neste trabalho.

Do ponto de vista dos valores culturais, Hofstede e Bond (1984) ressaltam que a importância em explorar este aspecto dá-se pelo fato de que a cultura não afeta apenas os processos psicológicos, mas também os sociológicos, políticos, econômicos e de sistemas sociais e que, por isso, compreender a sinergia entre valores e cultura é compreender como os fenômenos acontecem em uma determinada cultura, se ele é único ou ocorre e diversos locais. Por esta razão quanto mais se estuda valores culturais melhor se compreende tanto os processos gerais quanto os específicos.

Portanto, os estudos de valores culturais buscam evidências de universalismos, por meio de uma série de estudos paralelos feitos em diferentes nações, para explorar o significado de um conceito. Se achar um fenômeno que tem o mesmo correlato em diferentes contextos culturais, tem-se mais evidências 
de que se está estudando fenômenos equivalentes. Esta equivalência entre culturas pode encontrar resistência dentro de um contexto de análise da antropologia cultural, pois a primeira vista pode parecer considerar que a complexidade do fenômeno cultural seria estudada apenas por um conjunto de condições (BOAS, 2004), mas para os psicólogos transculturais esta é a melhor forma de verificar padrões comportamento (coincidentes ou dissonantes) em distintas partes do globo e, assim, trabalhar para poder melhorar a compreensão e interação entre as culturas, dentro da perspectiva de um mundo em constantes trocas culturais (SMITH et al, 2013).

$\mathrm{Na}$ busca destes universalismos o pesquisador Hofstede iniciou um trabalho relacionado aos valores do trabalho da IBM em 40 países. Ao final deste trabalho, o autor percebeu que poderia dividir as culturas conforme quatro dimensões culturais: masculinidade-feminilidade, evitação das incertezas, distância do poder, individualismo-coletivismo.

Masculinidade-feminilidade, refere-se às características tipicamente atribuídas aos sexos. 0 estudo revelou que os valores das mulheres diferem menos entre as sociedades que os valores dos homens e que os valores dos homens variam, de um país para outro, em conteúdos mais competitivos, bastante diferente dos valores das mulheres. O Polo da competitividade é chamado de masculino, enquanto o polo do cuidado de feminino. Esta dimensão evidencia a diferença entre os valores dos homens e das mulheres.

Evitação das incertezas indica o grau de tolerância de uma sociedade para com as incertezas e ambiguidades. Evidencia como a cultura programa seus membros para se sentirem desconfortáveis ou confortáveis em situações imprevistas. Culturas com alta evitação de incertezas buscam diminuílas com leis rígidas ou religiões/filosofias baseadas em verdades absolutas. Já as culturas com menor índice de evitação das incertezas, são mais tolerantes com opiniões diferentes, buscam ter o mínimo possível de regras, permitem a existência de correntes ideológicas opostas, a verdade é relativa.

A distância do poder guarda relação com a forma em que os indivíduos menos poderosos aceitam e esperam que o poder seja distribuído de forma desigual. Representa a desigualdade, porém é medida de baixo para cima, ou seja, o quão os menos poderosos (e os mais poderosos) aceitam essa desigualdade.

O Individualismo-Coletivismo se refere ao grau em que os indivíduos estão integrados em grupos. No individualismo esperam-se pessoas mais independentes da interferência grupal e que todos cuidem de si mesmos e de seus familiares mais próximos. Já no coletivismo esperam-se pessoas com metas grupais, integradas a grupos que os protege em troca de uma lealdade inquestionável. Nesta dimensão a noção de endogrupo (grupo ao qual o indivíduo pertence) e exogrupo (os demais grupos) é muito forte, pois para os coletivistas deve-se proteger o endogrupo e estimular a competição com o exogrupo.

De todas as quatro dimensões, aquela que é mais estudada e que os pesquisadores encontram melhores explicações para os fenômenos estudados é a dimensão individualismo-coletivismo.

Após o estudo original das dimensões culturais, foram feitas replicações em busca de maior validade para a teoria. Todavia em replicação do estudo na China, utilizando os valores chineses apenas 3 das 4 dimensões originais foram mantidas: individualismo-coletivismo, masculinidade-feminilidade e distância do poder. A dimensão evitação das incertezas não foi encontrada, sendo substituída pela dimensão orientação para longo prazo. Esta dimensão representa culturas que são orientadas para 
recompensas futuras, respeito pela tradição e preservação da face. Preservação da face diz respeito ao cuidado com a reputação do indivíduo.

Em outra replicação, desta vez na Rússia, Hofstede e Minkov (2010) encontram além das quatro dimensões originais mais duas dimensões: indulgência-restrição e monumentalismo-autodiminuição. $\mathrm{Na}$ indulgência a sociedade permite a gratificação livre de desejos e sentimentos (especialmente, com relação ao desejo e consumo); na restrição, há controle da gratificação, as pessoas se sentem menos possibilitadas a desfrutarem a vida. Monumentalismo representa culturas que recompensam as pessoas que são como monumentos, a sociedade é orgulhosa dos ícones daquela cultura. Na autodiminuição, uma sociedade recompensa a humildade e flexibilidade.

Triandis e Gelfland (1998) reconhecem a utilidade das dimensões propostas por Hosftede, porém argumentam que a dimensão mais estudada (individualismo-coletivismo) não deve ser vista como tão dicotômica como proposto originalmente. Os autores argumentam que tanto o individualismo quanto o coletivismo pode ser horizontal (enfatizando igualdade) ou vertical (enfatizando hierarquia) e que esta é uma distinção viável e importante. As maneiras pelas quais estas ênfases combinam com o individualismo e coletivismo produzir quatro padrões distintos: individualismo horizontal, individualismo vertical, coletivismo horizontal e coletivismo vertical.

No individualismo horizontal a cultura valoriza a diferenciação entra as pessoas, mas não baseada em competição e busca por maior status. Já o individualismo vertical valoriza a diferenciação entre as pessoas baseada no nível de status e de sucesso, existe valorização da competição entre as pessoas na busca pela hierarquia.

O coletivismo horizontal indica que as pessoas se percebem similares umas às outras, não é valorizada a submissão à autoridade, pois o poder está atrelado àqueles que representam a benevolência e ajuda ao outro. Por outro lado, no coletivismo vertical existe a submissão da pessoa aos objetivos do grupo, à autoridade. A competição entre endo e exogrupo e aceita e estimulada.

Por meio desta diferenciação horizontal/vertical, Triandis e Gelfland (1998) concluem que os individualistas verticais salientam a concorrência e o hedonismo ainda mais do que os individualistas horizontais; os individualistas horizontais salientam a autossuficiência. Os coletivistas verticais parecem ser mais autoritários e tradicionais, mas também se destaca a sociabilidade; os coletivistas horizontais salientam a sociabilidade, a interdependência e o hedonismo. Desta forma, os estudos que utilizam destas quatro relações podem investigar a como elas se relacionam a temas como atribuições, conformidade, persuasão, liderança, conflito e justiça, processos de grupo e gênero.

Como crítica a esta análise baseada em nações Schwartz (1992) argumenta que não se pode comparar os valores no nível nacional até que se tenha a estrutura dos valores no nível individual. Então sim, comparando os valores individuais em diferentes partes do mundo, pode-se produzir o mapeamento mundial dos valores. Para buscar esse mapeamento mundial de valores, Schwartz (1992) baseou-se, principalmente, no estudo de Rokeach (1973), mas também considerou outros trabalhos desenvolvidos na área de valores (BRAITHWAITE \& LAW, 1985; CHINESE CULTURE CONNECTION, 1987; HOFSTEDE, 1980; LEVY \& GUTTMAN, 1974; MUNRO, 1985). Assim, os valores encontrados foram elencados em um instrumento que fora traduzido em 13 línguas. A coleta de dados ocorreu em 20 países (Alemanha, Argentina, Brasil, China, Espanha, Estônia, Finlândia, Grécia, Holanda, Israel, Itália, Japão, Hong Kong, Nova Zelândia, Polônia, Portugal, Taiwan, Estados Unidos, Venezuela e 
Zimbábue), totalizando 9140 respondentes. Foram pesquisados participantes de 8 religiões (Budismo, confucionismo, ortodoxa, ortodoxa grega, judaísmo, protestantismo, católica romana e xintoísmo), além de respondentes ateus. Como resultado deste esforço, têm-se a proposição dos valores que foram considerados comuns em todos os países pesquisados. Knafo, Roccas, Sagiv (2011) em uma análise dos artigos acadêmicos publicados sobre o tema no Journal of Cross Cultural Psychologyentre 1993 e 2009 evidenciam que o estudo foi replicado em 70 países e que a estrutura (e o conteúdo) dos valores têm-se mostrado estável, mesmo entre culturas diferentes.

O cuidado que se deve ter ao estudar valores culturais é que não se pode ter certeza que as dimensões de variação cultural que têm sido identificadas são realmente as dimensões mais importantes. Elas podem ser apenas aquelas mais fáceis de identificar, especialmente por se correlacionarem com grandes atributos, como riqueza de uma nação (SMITH, 2005). Desta forma é comum fazer análises entre riqueza de nações e consumo, porém pouco se estuda da relação entre valores culturais e consumo de falsificações de produtos de moda.

\section{VALORES CULTURAIS E CONSUMO DE FALSIFICAÇÕES DE PRODUTOS DE MODA}

Nos estudos relevantes para o consumo, várias comparações entre sociedades individualistas e coletivistas têm apontado para diferenças importantes no conteúdo de recursos de publicidade, o processamento e capacidade de persuasão de apelos publicitários e os determinantes da intenção de compra dos consumidores.

Pesquisas recentes sugerem que o individualismo/coletivismo pode coexistir na memória, de tal forma que os fatores contextuais podem temporariamente ativar tanto o self independente quanto o self interdependente. Estas ativações parecem alterar a percepção social e julgamentos de consumo de forma que são altamente consistentes com os valores culturais (SHAVIT, ZHANG \& TORELLI, 2009). A distinção cultural fornece evidências para o seu valor como preditor de novos fenômenos de psicologia do consumidor e como uma base para refinar a compreensão dos fenômenos conhecidos (SHAVITT et al., 2006).

Os consumidores usam determinados produtos ou marcas para expressar aos outros os seus valores pessoais (RICHINS, 1994). Embora a função de se auto expressar por meio de produtos pode refletir um objetivo universal, pesquisas recentes sugerem que determinadas culturas valorizam a auto expressão mais do que os outros fazem. Além disso, marcas e produtos variam em seu papel de auto expressão (SHAVITT, 2010), ou seja, algumas marcas são mais icônicas do que outras de forma que essas marcas podem ser mais susceptíveis de transportar e ativar significados culturais.

Shavit e Koo (2010) sugerem que as representações mentais de poder em termos de status e competição versus benevolência diferem de forma confiável entre origens e orientações culturais verticais e horizontais e, ainda, que os produtos que são orientados para hierarquia, luxo, prestígio e distinção eram geralmente mais prevalentes em sociedades que se presume terem perfis culturais verticais (por exemplo, Coréia, Rússia) do que um perfil cultural horizontal (Dinamarca).

Assim, a influência do individualismo/coletivismo não se dá apenas no ato do consumo, as empresas devem estar atentas a estes valores culturais no ato de criar anúncios acerca dos produtos. A maioria das pesquisas sobre as influências culturais sobre o julgamento e persuasão examinou a implicações do individualismo-coletivismo. Em geral, os resultados sugerem que a prevalência ou a persuasão de 
um determinado tipo de recurso coincide com a orientação de valor cultural da sociedade (SHAVITT, LEE, E TORELLI, 2006). Os autores sugerem ainda que a cultura pode afetar a forma como as pessoas processam e interpretam informações relacionadas ao produto. No entanto, as características de marcas e produtos podem restringir o papel das variáveis culturais no processamento de informação e persuasão; algumas marcas e produtos servem como portadores de valores culturais mais fortes do que outras (SHAVITT, TORELLI E WONG, 2009).

A dificuldade de utilizar modelos culturais aplicados a consumo é que se deve primeiramente investigar qual a dimensão é importante para o que se busca, e só após isso é que se podem buscar medidas individuais sobre a dimensão investigada.

Nos últimos anos, a pesquisa em comportamento do consumidor abordou um conjunto ampliação das questões e dimensões culturais. Estas pesquisas têm propiciado uma melhor compreensão das relações entre cultura e autoimagem, motivação, estilo de pensamento, e de persuasão do consumidor. As pesquisas também começaram a abordar os mecanismos psicológicos subjacentes às diferenças transculturais nos julgamentos de consumo, e os produtos e contextos para que essas diferenças são mais propensas a serem observadas (SHAVIT \& KOO, 2010).

Allen (2000) sugeriu que o significado do produto é o que dá valor ao mesmo. Dois grandes tipos de sentido podem ser atribuídos a um produto: o significado utilitário e o simbólico. O significado utilitário representa a função concreta do produto, seus atributos, diz respeito a parte tangível de um objeto. O significado simbólico é o resultado de experiências sociais (através de instituições sociais, sistemas de comunicação e a cultura) que levam à categorização subjetiva do produto. Portanto o significado simbólico pode ser entendido como tendo relação direta com a cultura (ALLEN, NG \& WILSON, 2002; TORRES \& ALLEN, 2009; TORRES \& PEREZ-NEBRA, 2007; ALFINITO \& TORRES, 2012). Estes estudos evidenciam, ainda, que países mais individualistas tendem a fazer uma análise utilitária dos produtos e países mais coletivistas tendem a optar por escolhas mais simbólicas.

A moda deve ser vista como um fenômeno complexo em multinível, pois afeta as pessoas tanto em seus comportamentos individuais quanto nos comportamentos sociais. Assim, as decisões de consumo nesta área são baseadas pelo desejo de estar na moda e também por necessidade de pertencimento à grupos (SIMMEL, 1904). É um mercado que tende a trabalhar dentro de um princípio de prestígioexclusividade onde altos preços criam grande demanda e de efeito esnobe onde preços mais baixos reduzem a demanda (SOLOMON, 2011).

Os produtos de moda, em sua maioria são considerados uma forma de consumo conspícuo (ARIELY \& NORTON, 2009). O consumo conspícuo é aquele que além de satisfazer necessidades básicas também sinaliza para nós e para os outros as nossas crenças, nossas atitudes e identidades sociais. O consumo de produtos de moda (ainda que falsificados) é uma expressão clara de consumo conspícuo, pois paga-se o preço para suprir uma necessidade física, mas também para demonstrar status perante a sociedade. Ariely e Norton (2009) defendem que este tipo de consumo é o casamento perfeito entre o simbólico e o utilitário e que as pressões sociais aumentam a busca por este tipo de produto.

Para que um produto funcione como um símbolo ele deve ter afinidade de sentido entre os consumidores (ELLIOTT, 1994) e a cultura cria estes significados e os desloca pela sociedade até os consumidores (MCCRACKEN, 1986). Um sistema de produção de cultura (SPC) é o conjunto de 
indivíduos e organizações responsáveis pela criação de um produto cultural (SOLOMON, 2011). Na perspectiva da produção de cultura, a moda tem um papel importante, pois ela é uma forma de capturar e mover o significado cultural (DIAMOND, 2005; MCCRACKEN, 1986; SIMMEL, 1904). Moda "é o processo de difusão social pelo qual um novo estilo é adotado por alguns grupos de consumidores" (SOLOMON, 2011, p.617). Embora as pessoas tendam a igualar moda com vestuário (roupa), é importante observar que os processos de moda afetam todos os tipos de fenômenos culturais, o que inclui música, arte, arquitetura (SOLOMON, 2011). Pode-se pensar na moda como um código ou linguagem que nos ajuda a decifrar significados (ECO, 1976).

O perfil do consumidor de produtos de moda não é único e pode ser explorado por diversas facetas, por isso o comportamento do consumidor de moda é um vasto campo de pesquisas, pois exemplificam a subjetividade individual e a influência da cultura sobre o ser humano (BERRY, 1994; TWITCHELL, 2002). São ligados à símbolos da cultura material, político e social. O produto falsificado se torna um produto de luxo porque traz consigo a competência exigida para julgar bens e serviços de informação (KAPFERER, 2003). O encantamento trazido pelos produtos de moda deslumbra todas as classes sociais, em diversos países do mundo. A indústria chega a níveis de perfeição que os produtos se tornam obras de arte para se usar (SILVERSTEIN \& FIRSKE, 2005).

Em pesquisas sobre o tema das falsificações de produtos Tom, Garibaldi, Zeng e Pilcher (1998) encontraram o fator econômico como determinante para a compra de falsificações de luxo, porém outras pesquisas demonstram que consumo de falsificações de luxo ocorre por motivações sociais (WILCOX, KIM \& SEM, 2008) ou hedônicas (YOO \& LEE, 2009). No Brasil Terres, Cavedon e Santos (2010) afirmam que consumo de falsificações de luxo não é feito por consumidores de baixa renda e sim classe média. Já Bian e Moutinho (2011) encontraram fraca correlação entre fatores demográficos e consumo de falsificações de luxo. Alfinito e Torres (2012) evidenciam que o consumo não se dá pelo produto apenas (distante e frio), mas sim pelo o que ele significa. Ayrosa (2007) ressalta em sua pesquisa que o valor mais destacado para os consumidores de falsificações foram prazer, seguido de status, eficiência e altruísmo. Importante ressaltar que os estudos não são focados na percepção de valor dos consumidores, mas somente do ato de consumir.

Wilcox, Kim e Sen (2008) demonstraram que o desejo dos consumidores por marcas de moda falsificada depende das motivações sociais. Em particular, os autores mostram que a preferência dos consumidores por marcas falsificadas é maior quando a marca de luxo tem uma função de valor expressivo (os autores usam o termo valor expressivo para referir-se às marcas que são vista como de alto valor no mercado). Assim, as crenças morais dos consumidores sobre o consumo de falsificados afetam suas preferências por falsificações somente quando as marcas de luxo têm uma função de valor expressivo. Finalmente, os autores demonstram que as funções sociais das marcas de luxo podem ser influenciadas por elementos do mix de marketing (design do produto, publicidade), permitindo que os comerciantes reduzam a demanda por marcas falsificadas por meio de ações específicas de marketing mix. Todavia Yoo e Lee (2009) defendem que não é apenas modificando aspectos ligados ao marketing que se consegue combater as falsificações, que é preciso investigar o processo muito além do mundo corporativo.

No mercado da falsificação, o mundo da moda é o maior alvo (MERAVIGLIA, 2015). Os itens mais buscados são: acessórios, relógios, sapatos e roupas. A autora defende que neste setor, mais do que em qualquer outro, o grande responsável pelo sustento do fenômeno é o consumidor. E não se trata apenas do consumidor com baixo poder aquisitivo, o que se percebe é que um número significativo 
de consumidores com alto poder aquisito busca produtos falsificados (IACC, 2010). As marcas de produtos de luxo investem uma alta quantia de verba para o combate às falsificações: a Cartier investiu no ano de 2005 cerca de $€ 3$ milhões e a Louis Vuitton $€ 12$ milhões (MERAVIGLIA, 2015). Esta última marca criou um setor com mais de 60 pessoas dedicadas especificamente ao estudo, investigação e combate das falsificações. Apesar dos esforços, os consumidores não parecem entender a real natureza do problema, pois pesquisas mostram que grande parte deles já fizeram a compra e/ou uso por mais de uma vez e não demostram intenção de parar de fazê-lo (IACC, 2010; MERAVIGLIA, 2015).

Yoo e Lee (2009) inspirados no trabalho de Wee et al. (1995) investigaram os antecedentes não monetários especificamente para consumo de produtos de moda de luxo falsificados e obtiveram os seguintes resultados: a intenção de compra de falsificações de moda de luxo foi previsto positivamente por experiências passadas de compra de falsificações, atitudes positivas em relação à compra de falsificações por benefícios econômicos, atitudes positivas para a compra de falsificações por benefícios hedônicos e do materialismo. Além disso verificaram que a intenção de compra de produtos falsificados foi positivamente relacionada à intenção de compra de originais, mas a intenção de compra de originais foi negativamente relacionada à intenção de compra de falsificações.

Phau e Teah (2009) investigaram como os fatores sociais (suscetibilidade informacional, suscetbilidade normativa e coletivismo) e pessoais (conciência, busca por novidades, integridade, gratificações pessoais e consumo de status) influenciam a formação de atitudes perante os produtos de luxo falsificado na China. Os resultados apontam que a susceptibilidade normativa influencia de forma mais importante a atitude do consumidor perante as falsificações e, consequente, intenção de compra. Os consumidores são mais influenciados pelas atitudes perantes as falsificações de luxo do que por considerações éticas e legais. Não foi encontrada relação entre o coletivismo, gratificação pessoal ou busca por novidades e o consumo de falsificados. Integridade e consumo de status foram os fatores mais importantes que influenciam às atitudes e a intenção de compra dos consumidores. Como forma de combate à falsificação os autores sugerem que a achave para isso pode estar na mudança de atitudes dos consumidores.

Em um estudo feito em Dubai, Fernandes (2013) investigou as variáveis consciência de moda, normas subjetivas, julgamento ético, consciência, e autoidentidade (renomeada para autoambiguidade). Norma subjetiva apresenta-se correlacionada à consciência de moda dado que a moda é mais sobre o estilo aceitável (do ponto de outras pessoas de vista). Julgamento ético, consciência, autoambiguidade e normas subjetivas são significativamente correlacionados com a intenção de compra de falsificados. É ressaltada a importância de se estudar o papel do grupo, pois os dados sugerem que quanto maior a pressão grupal, maior a tendência de consumo dos produtos falsos. Um achado não esperado nesta pesquisa foi o de que consciência de moda não se apresentou relacionado com a probabilidade de compra de falsificados como era esperado. $\mathrm{O}$ autor sugere que esta variável seja melhor investigada em uma amostra que não seja composta majoritariamente por estudantes e acredita que ela pode ser influenciada de forma diferentes de acordo com cada cultura.

Em um estudo recente Zampetakis (2014) investigou quais emoções são sentidas pelos consumidores de produtos de luxo falsificados. Trata-se de um estudo exploratório, porque apesar da relação entre emoções e consumo já ter sido verificada em outros contextos, o autor é pioneiro ao fazê-lo com relação aos produtos de luxo falsos. Foram investigas 8 emoções: orgulho, culpa, vergonha, medo, raiva, aflição, interesse e alegria. Os resultados indicam que durante o consumo de protudos 
falsificados não existe predominância de uma emoção, mas sim de um mix delas, sendo as emoções de culpa e vergonha as que foram relatadas como as mais intensas. $O$ autor salienta que apesar das duas emoções negativas aparecerem como sendo as mais fortes, ainda são relatadas que ocorrem ligadas a elas emoções positivas como alegria e interesse. $E$, ainda, essas emoções positivas tendem a ser mais relatadas por participantes que têm atitudes favorável ao consumo de falsificações.

\section{CONSIDERAÇÕES FINAIS}

O que se percebe é que quanto mais vertical a cultura, mais valorizado é o consumo como forma de diferenciação social. Já que os produtos de luxo são caros em termos absolutos (monetários) e relativos (o que representam), eles proporcionam uma boa forma de gerenciamento de impressão (GOFFMAN, 1959). E se o consumidor não pode arcar com os preços cobrados, mas busca a satisfação do desejo pelas perspectivas hedônicas, ele é levado a crer que produtos originais e falsificados são similares, uma vez que as falsificações recebem elementos da identidade (nome, símbolo e cor) das marcas dos produtos originais (ZAICHKOWSKY, 1995) e o consome.

O crescimento da falsificação no Brasil é notório (Brasil, 2006) e os níveis de qualidade e similaridade interferem na decisão do consumidor já que este pode não estar apto a diferenciar o produto falso do original. O consumidor pode (nondeceptive counterfeit) ou não (deceptive counterfeit) demonstrar um conhecimento específico ao escolher uma falsificação (STREHLAU, 2004). Seja por qual maneira se dê o consumo o que se busca é que a imagem seja mostrada como de alguém que pertence aquele grupo e utiliza os mesmos símbolos daquela cultura. Funciona como um camaleão que se transforma para adaptar-se às diversas situações a quem é submetida (BAUMEISTER \& FINKEL, 2010). Importante ressaltar que o que é prestigioso para um grupo social pode não ser para outro, pois não é apenas a marca que dá significado à distinção social, mas é o conjunto do símbolo com os outros valores culturais e artefatos daquela cultura.

Compreender as diferenças culturais tornou-se crucial para uma comunicação eficaz com o consumidor. Em pesquisas futuras, será importante distinguir ainda mais semelhanças culturais, as diferenças nos julgamentos dos produtos consumidos, identificar semelhanças e diferenças intranacionais, investigar se existe relação entre densidade populacional das cidades e a influência da cultura no consumo e o consumo como ferramenta de gerenciamento de impressão (BAUMEISTER \& FINKEL, 2010).

Metodologicamente, os pesquisadores devem buscar explicar o papel dos valores culturais no consumo de falsificações por meio de desenhos experimentais, pois desta forma buscar-se-ão relações de causalidade e não apenas correlações entre os fatores. Além disto, os estudos correlacionais devem buscar melhores instrumentos de forma que consigam aumentar a explicação da significância, pois atualmente os estudos de valores pouco da variância é explicada. Muitos são os fatores que influenciam uma cultura, mas os pesquisadores devem continuar a investigar tantos grupos quanto possível para contribuir para uma melhor compreensão dos padrões de preferência do produto em diferentes culturas. 


\section{REFERÊNCIAS}

AJZEN, I. Consumer Attitudes and Behavior. In Handbook of consumer psychology. New York: Psychology Press, 2008. 525-548.

ALLEN, M. W. (2000). The attribute-mediation and product meaning approaches to the influences of human values on consumer choices. In Advances in psychology research. Huntington: Nova Science, 2000. 31-76

ALLEN, M.; NG, S.; WILSON, M. The functional approach to instrumental and terminal values and the value-attitude behavior system. European Journal of Marketing, 36, 111-135. 2002

ALFINITO, S.; TORRES, C. V. Modelo de influência cultural no consumo :uma proposta baseada em axiomas sociais, Rev Adm Mackenzie. São Paulo, V. 13, N. 5, 15-38. 2012

ARIELY, D.; NORTON, M. I. Conceptual Consumption. Annual Review of Psychology, v. 60, n. 1, p. 475-499, jan. 2009.

AYROSA, Eduardo. A falsa ilusão de ter: investigando os tipos de valores para o consumidor de falsificação. Dissertação de mestrado, FGV, 2009.

BRAITHWAITE, V.; LAW, G. Structure of human values: Testing the adequacy of the Rokeach Value Survey. Journal of Personality and Social Psychology. Vol 49, 1985.

BAUMEISTER, R; FINKEL, J. Advanced Social Psychology: the state of the science. New York: Oxford University Press, 2010.

BERRY, C. The Idea of Luxury: A Conceptual and Historical Investigation, Cambridge: Cambridge University Press, 1994.

BIAN, X.; MOUTINHO, L. The role of brand image, product involvement, and knowledge in explaining consumer purchase behaviour of counterfeits: Direct and indirect effects. European Journal of Marketing, Vol. 45, 2009.

BOAS, Franz. Antropologia cultural. Org. Celso Castro. Rio de Janeiro: Jorge Zahar, 2004.

BRASIL. Ministério da Justiça. Apreensões de produtos piratas bate recorde em 2006. Brasília, 2006.

CHINESE CULTURE CONNECTION. Chinese values and the search for culture-free dimensions of culture. Journal of Cross-Cultural Psychology, Vol 18, 1987.

CHISTOVÃO, D. Propriedade industrial: a confusão de sinais distintivos é objeto de ação judicial. Valor Econômico, 2001.

DELENER, N. International counterfeit marketing: success without risk. Review of Business, Volume Primavera, 16-19. 2000.

DIAMOND, E. The Fashion Consumer: Identification and Analysis. Fashion Retailing: A MultiChannel Approach, p. 71-96, 2005.

ECO, U. A Theory of Semiotics. Bloomington: Indiana University Press, 1976. 
ELLIOTT, R. Exploring the Symbolic Meaning of Brands. British Journal of Management, v. 5, n. 2, p. 13-19, 1994.

FERNANDES, C. Analysis of counterfeit fashion purchase behaviour in UAE. Journal of Fashion Marketing and Management: An International Journal, 17(1), 85-97, 2013.

GENTRY, J. The legitimacy of counterfeits: consumers choosing counterfeit brands and tourist seeking authentic counterfeits. In Macromarketing Conference. Sydney. 2002

GOFFMAN, Erving. The Presentation of Self in Everyday Life. Nova Iorque: Anchor Books, 1959

GRANSTRAND, O. Innovation and intellectual property rights. In The oxford handook of innovation. Oxford: Oxford University Press. 2005.

GROSSMAN, G; SHAPIRO, C. Counterfeit-product trade. The American Economic Review, XX(i), 50-75, 1988

HEINE, S. J. Cultural psychology. In Advanced Social Psychology. Oxford: Oxford University Press, 2010.

HOFSTEDE, G.; BOND, M. H. Hofstede's Culture Dimensions: An Independent Validation Using Rokeach's Value Survey. Journal of Cross-Cultural Psychology, 15(4), 417-433, 1984.

HOFSTEDE, G; MINKOV, M. Culture and Organizations: Software of the mind: intercultural cooperation and its importance for survival. McGraw-Hill, 2001.

JACOBY, J; KAPLAN, L. The components of perceived risk. Third Annual Conference of Association for Consumer Research, II(a), 382-393, 1972.

KAPFERER, J. As Marcas capital da empresa: criar e desenvolver marcas fortes. Porto Alegre: Bookman, 2003.

KLUCKHOHN, C. Values and value-orientations in the theory of action: An exploration in definition and classification. In Toward a general theory of action. Cambridge: Harvard University Press, 1951.

KNAFO, A; ROCCAS, S; SAGIV, L. The values in cros-cultural research: a special issue in honor of shalom Schwartz. Journal of Cross-Cultural Psychology, Vol 42, 2011.

LEVY, S., \& GUTTMAN, L. Values and attitudes of Israeli high school youth. Jerusalem: Israel, 1974

MCCRACKEN, G. Culture and Consumption: A Theoretical Account of the Structure and Movement of the Cultural Meaning of Consumer Goods. Journal of Consumer Research, v. 13, n. 1, p. $71,1986$.

MERAVIGLIA, L. Counterfeiting, fashion and the civil society. Journal of Fashion Marketing and Management: An International Journal, 19(3), pp.230-248, 2015.

MOOIJ, M. D; HOFSTEDE, G. Cross-Cultural Consumer Behavior : A Review of Research Findings. 181-192. 2001. 
MUNRO, D. A free-format values inventory: Explorations with Zimbabwean student teachers. South African Journal of Psychology, Vol 15, 1985.

PHAU, I.; TEAH, M. Devil wears (counterfeit) Prada: a study of antecedents and outcomes of attitudes towards counterfeits of luxury brands. Journal of Consumer Marketing, 26(1), 1527, 2009.

RICHINS, M. L. Special possessions and the expression of material values. Journal of Consumer Research, 21(3), 522-531, 1994.

ROHNER, R. P. Toward a conception of culture for crosscultural psychology. Journal of Cross Cultural Psychology, 15, 111-138, 1984

ROKEACH, M. The nature of human values. New York: Free Pres, 1973

SCHWARTZ, S. H. Universals in the content and structure of values: theoretical advances and empirical tests in 20 countries. Advances in Experimental Social Psychology, 25, 65, 1992.

SHAVITT, S.; LALWANI, A.K.; ZHANG, J.; TORELLI, C.J. The horizontal/vertical distinction in cross-cultural consumer research. Journal of Consumer Psychology, 16 (4), 325-356, 2006.

SHAVITT, S.; TORELLI, C; WONG, J. Identity based motivation in a consumer context. Journal of Consumer Psychology, 19 (3), 261-266, 2009.

SHAVITT, S.; KOO, Minkyung. Cross-cultural psychology of consumer behavior. London: Wiley, 2010

SHERIF, M. The psychology of social norms. New York: Harper \& Row, 1936.

SILVERTEIN, M.; FIRSKE, N. Trading up: why consumers want new luxury goods - and how companiel create them. London: Penguin Group, 2005.

SIMMEL, G. Fashion. International Quarterly, v. 10, n. 1, p. 130-155, 1904.

SMITH, P., BOND, M; KAGITÇIBASI, C. Understanding Social Psychology Across Cultures: Living and working in a changing world. London: Sage Publications, 2006.

SMITH, P; FISCHER, R; VIGNOLES, V; BOND, H. Understanding Social Psychology Across Cultures: Engaging with others in a changin world. London: Sage Publications, 2013.

SOLOMON, M. Comportamento do consumidor. 9a. ed. Porto Alegre: Bookman, 2011.

STREHLAU, Suzane. Marketing do Luxo. São Paulo. Cengage Learning, 2008.

SUMMER,W. Folskways. Boston: Ginn, 1906.

TERRES, M.; CAVEDON, R.; SANTOS, C. O que reluz definitivamente não é ouro. Estudos sobre o consumo de réplicas de marcas de luxo. Ciências sociais em perspectiva, Vol. 9, 2010.

TOM, G.; GARIBALDI, B.; ZENG, Y.; PILCHER, J. Consumer Demand for Counterfeit Goods. Psychology And Marketing, Vol.15, 1998 
TORRES, C., Pérez-NEBRA, A. The Influence of Human Values on Holiday Destination Choice in Australia and Brazil, 63-76, 2007

TORRES, C.; ALLEN, M. Valores Humanos e Escolha do Consumidor na Austrália e Brasil, 25, 611-620, 2009.

TRIANDIS, $\mathrm{H}_{.} ;$GELFAND, $\mathrm{M}$. Converging measurement of horizontal and vertical individualism and collectivism. Journal of Personality and Social Psychology, 74(1), 118-128, 1998.

TWITCHELL, J. Living it up: our love affair with luxury. New York: Columbia university press, 2002

WILCOX, K; KIM, H; SEN, S. Why do consumers buy counterfeits luxury brands. Journal of marketing research, Vol. XLV, 2008.

YOO, B; LEE, S. Buy genuine fashion products of counterfeits?. Advances in consumer research, Vol. 36, 2009.

ZAICHKOWSKY, J. DEFENDING YOU BRAND AGAINST IMITATION. Westport: Quorum, 1995.

ZAMPETAKIS, L. The emotional dimension of the consumption of luxury counterfeit goods: an empirical taxonomy. Marketing Intelligence \& Planning, 32(1), 21-40, 2014. 